\title{
Bronchial Artery Aneurysm as a Cause of Atelectasis
}

\author{
Mikio Oka, Minoru Fukuda, Kenji Terashi, Hiroshi Takatani, Fumihiko Narasaki, \\ Reiji Nakano, Junji Tsurutani, Yo-ichi Nakamura, Takashi Kasai, Seiji Nagashima, \\ Yuji Noguchi, Hiroshi Soda and Shigeru KoHNO
}

\begin{abstract}
A 54-year-old nonsmoker female developed atelectasis of the anterior basal segment of the right lower lobe. A non-pulsating endobronchial tumor was observed bronchoscopically obstructing the right basal bronchus. The tumor was confirmed on arteriography to be a saccular aneurysm of the right bronchial artery. The aneurysm was treated with bronchial artery embolization. Bronchial artery aneurysm, without a predisposing disease, is quite rare, but should be considered as an etiological factor of atelectasis.
\end{abstract}

(Internal Medicine 36: 917-919, 1997)

Key words: bronchial tumor, arterial embolization, bronchoscopy, vascular lesion

\section{Introduction}

Bronchial artery aneurysm (BAA) is a rare disease classified into intrapulmonary and mediastinal types (1). BAA is occasionally discovered on angiography in patients with hemoptysis (1). Except in a few cases associated with bronchiectasis, tuberculosis, fungal infections or cystic fibrosis (2-5), the clinical features and etiologic factors of BAA are unknown. We present a case with BAA mimicking an endobronchial tumor and causing atelectasis. The aneurysm was treated successfully with arterial embolization.

\section{Case Report}

A 54-year-old nonsmoker female was admitted in September 1994 to Nagasaki University Hospital for further evaluation of a bronchial tumor in the right basal bronchus. The patient presented three months earlier with fever (approximately $39^{\circ} \mathrm{C}$ ) and cough. Infiltration in the right anterior basal segment was detected on a chest radiogram and computed tomogram in a local hospital (Fig. 1). The white blood cells (WBC) count and C-reactive protein (CRP) were $9,200 / \mu 1$, with neutrophilia, and $13.2 \mathrm{mg} / \mu \mathrm{l}$ (normal value $<0.17 \mathrm{mg} / \mu \mathrm{l}$ ), respectively. After admission to the local hospital, systemic antibiotics were administered for one week based on a provisional diagnosis of bacterial pneumonia. While the clinical signs and symptoms, leukocytosis, and elevated CRP improved, atelectasis of the anterior basal segment was still present on chest radiograms. Bronchoscopy was performed to explore the cause of atelecta- sis, and a solitary endobronchial tumor thinly covered with blood was detected, almost occluding the entire right basal bronchus. At that time, no further procedure was performed to confirm the diagnosis in order to avoid life-threatening bleeding.

On transfer to our hospital, there were no abnormal findings on physical examination apart from the presence of diminished respiratory sounds in the right lower lung field latero-posteriorly. Atelectasis was still detected on the chest radiogram. The blood

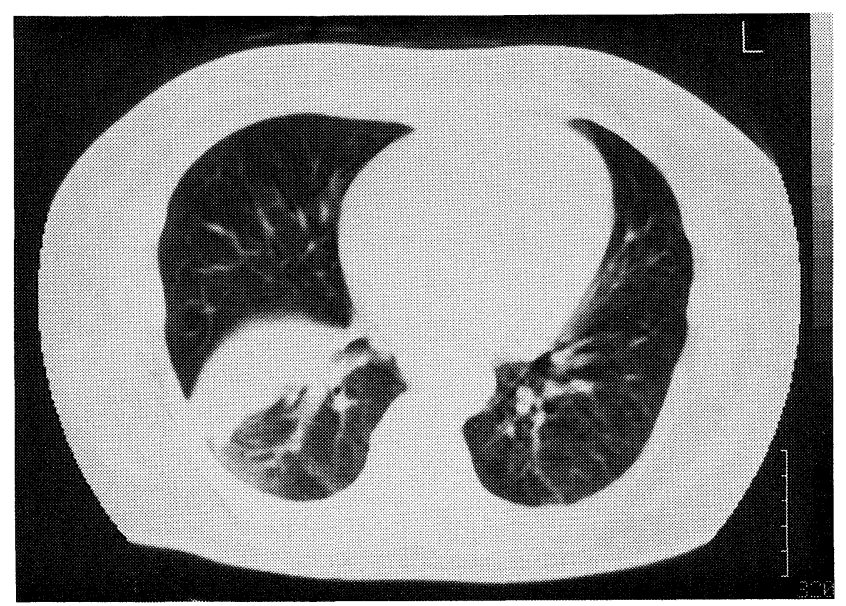

Figure 1. A computed tomography showing atelectasis of the anterior basal segment of the right lower lobe.

From the Second Department of Internal Medicine, Nagasaki University School of Medicine, Nagasaki

Received for publication January 23, 1997; Accepted for publication September 19, 1997

Reprint requests should be addressed to Dr. Mikio Oka, the Second Department of Internal Medicine, Nagasaki University School of Medicine, 1-7-1 Sakamoto, Nagasaki 852 


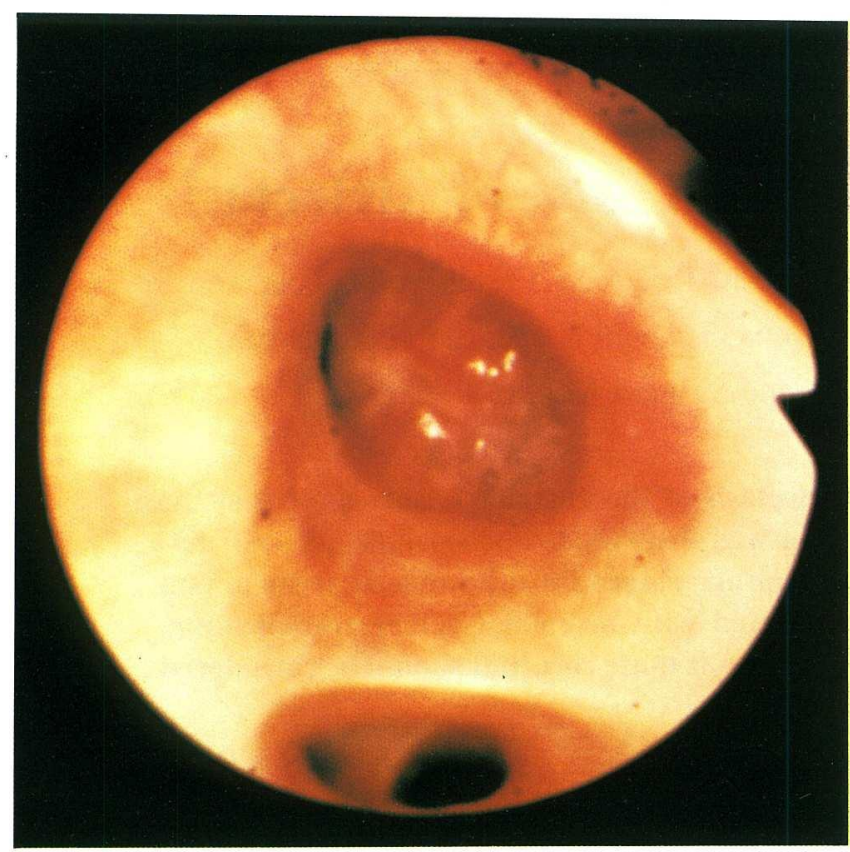

Figure 2. Bronchoscopic view of the right basal bronchus showing an aneurysm resembling an endobronchial tumor.

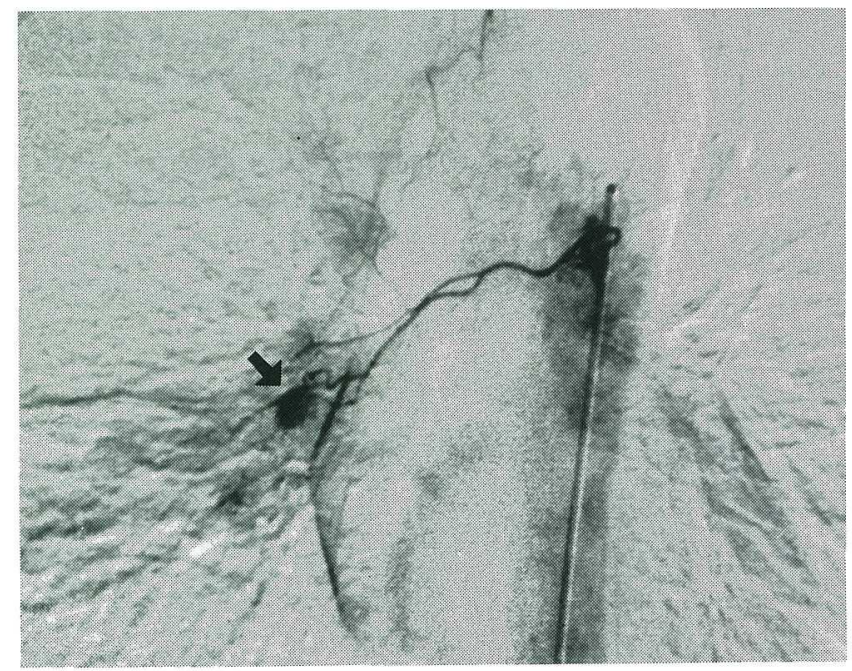

Figure 3. Digital subtraction angiogram of the right bronchial artery showing a solitary saccular aneurysm approximately $1.0 \mathrm{~cm}$ in diameter (arrow), in an intrapulmonary portion.

cell count, biochemical and blood gas analyses were all normal, but CRP was only marginally increased $(0.43 \mathrm{mg} / \mu \mathrm{l})$. Cytology, bacteria, fungi and acid-fast bacilli on sputum were all negative. At another bronchoscopic examination, the endobronchial tumor in the right basal bronchus was suspected to be a vascular lesion by the easy pitting of the tumor using a polyethylene tube inserted through the bronchoscopy channel, although pulsation was not observed (Fig. 2). Confirmation that the mass was a bronchial artery aneurysm was established by a

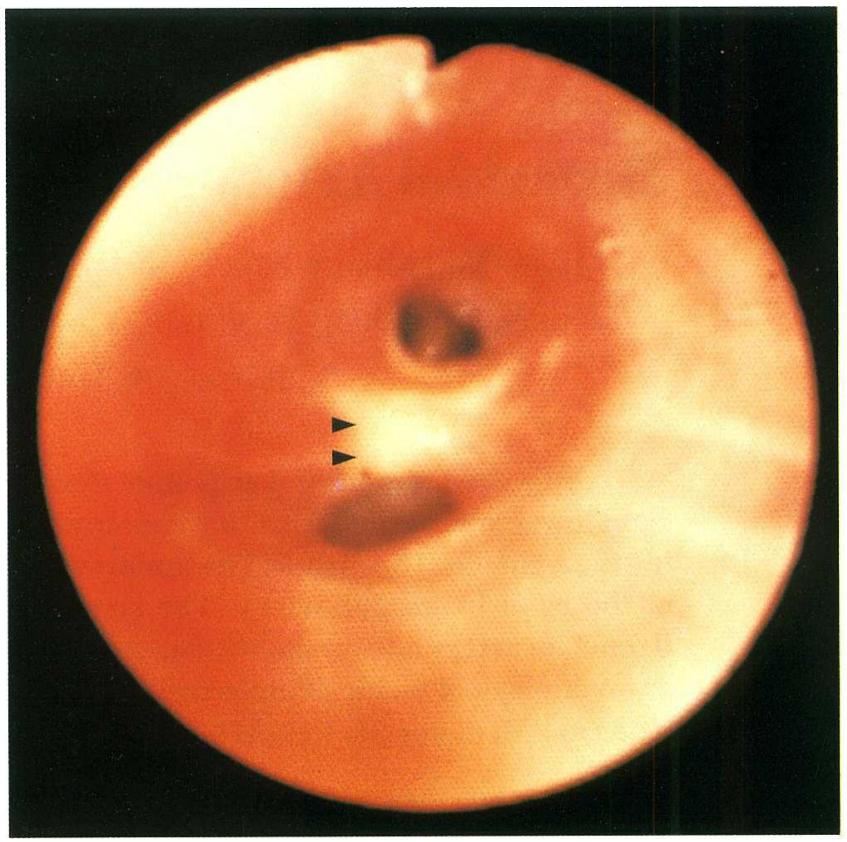

Figure 4. Bronchoscopic findings in the right basal bronchus approximately one month after bronchial artery embolization. The endobronchial mass completely disappeared. The white spot (arrow) suggests that the aneurysm had existed in the spur for some time, between the anterior and latero-posterior basal bronchus.

right bronchial arteriogram, in which the aneurysm was found to correspond with the tumor (Fig. 3). The aneurysm was treated with bronchial artery embolization using gelatin sponge (Gelfoam; Upjohn Kalamazoo, MI, USA). The tumor completely disappeared one month later as confirmed bronchoscopically (Fig. 4). The findings on bronchoscopic examination suggested that the aneurysm existed for some time in the submucosal region of the spur between the right anterior and latero-posterior basal bronchus (Fig. 4). At present, the aneurysm has not regrown after embolization.

\section{Discussion}

Bronchial artery aneurysm is extremely rare in patients without a predisposing pulmonary or systemic disease, such as chronic infection or Rendu-Osler-Weber syndrome (1). The present patient did not have any predisposing disease, thus allowing the diagnosis of a BAA. BAAs are occasionally discovered on a routine investigation of hemoptysis, which could, at times, be life-threatening $(1,2)$. To our knowledge, no BAA has been reported to cause atelectasis, although the size of BAA may vary from a few millimeters in diameter to a large size $(1,3,6)$.

The BAA of the present patient without hemoptysis resembled a solitary endobronchial tumor, and resulted in atelectasis. Although an arterial lesion in the bronchus is usually seen bronchoscopically as a pulsating mass or as a reddish swelling 


\section{Bronchial Artery Aneurysm}

covered with a normal bronchial mucosa (4), a differential diagnosis from an endobronchial tumor is sometimes difficult. Before bronchoscopic forceps biopsy or brushing, pitting the mass carefully by a tube inserted through the bronchoscopic channel may be useful in differentiating between an aneurysm and a tumor due to the different consistency of the two masses. This is essential in order to avoid possible life-threatening bleeding. Furthermore, bronchoscopists should recognize a bronchial artery aneurysm as a cause of atelectasis.

Arterial embolization of bronchial, mammary or intercostal arteries is a standard treatment for massive or recurrent hemoptysis (7). The results are favorable even at long-term follow-up although relapse may occur in some patients. While our patient was successfully treated with embolization, aneurysm may potentially re-enlarge or reappear multifocally following embolization. Therefore, we feel that bronchoscopic and radiologic follow-up studies are necessary. The clinical features of bronchial artery aneurysm, without predisposing diseases, should be further clarified by collecting more data on such patients.

\section{References}

1) Osada H, Kawada T, Ashida H, Sodemoto Y, Noguchi T. Bronchial artery aneurysm. Ann Thorac Surg 41: 440, 1986.

2) Hall RJC, Miller GAH, Kerr IH. Ruptured bronchial artery aneurysm mimicking aortic dissection. Br Heart J 39: 909, 1977.

3) Servois V, Denys A, Sibert A. Mycotic aneurysm of the bronchial artery: a rare cause of hemoptysis. Am J Roentgenol 159: 428, 1992 (Letter).

4) Katoh O, Yamada H, Hiura K, Nakanishi Y, Kishikawa T. Bronchoscopic and angiographic comparison of bronchial arterial lesions in patients with hemoptysis. Chest 91: 486, 1987.

5) Remy-Jardin M, Remy J, Ramon PH, Fellous G. Mediastinal bronchial artery aneurysm: dynamic computed tomography appearance. Cardiovasc Intervent Radiol 14: 118, 1991.

6) Shaer AH, Bashist B. Computed tomography of bronchial artery aneurysm with erosion into the esophagus. J Comput Assist Tomogr 13: 1069, 1989.

7) Roberts AC. Bronchial artery embolization therapy. J Thorac Imaging 5: 60, 1990. 